\title{
INFLUENCIA DE LA FUENTE DE POLEN Y SU EFECTIVIDAD EN LA CALIDAD DE FRUTOS DE CHIRIMOYA (Annona cherimola MILL.)
}

\author{
Isela Apolonio*, Álvaro Castañeda ${ }^{1 / * *}$, Omar Franco ${ }^{* *}$, Edgar Jesús Morales ${ }^{* *}$, Andrés González, \\ Palabras clave: Polinización; fuente de polen; amarre de fruto; anonáceas. \\ Keywords: Pollination; pollen source; fruit set; Anonaceae. \\ Recibido: $13 / 08 / 14$ \\ Aceptado: 07/01/15
}

\section{RESUMEN}

Se evaluó la influencia de la fuente de polen y los horarios de polinización sobre el amarre y la calidad de frutos en 3 cultivares de chirimoya: 'Campas', 'White' y 'Bonita'. El cultivar 'White' se cultiva comercialmente en California, mientras que 'Campas' y 'Bonita' son cultivados, en menor escala, en España. Se realizó autopolinización y polinización cruzada artificial sin mezcla de polen en cada cultivar, en 3 horarios diferentes (07:00-09:00, 10:00-12:00 y 18:00-20:00 h), empleando la técnica del pincel. En todos los cultivares, el porcentaje de amarre de frutos con polinización natural fue bajo (de 5 a 10\%). El tratamiento de autopolinización fue el menos eficiente, especialmente en 'Bonita' $(47,90 \%)$. Por el contrario, cuando 'Bonita' fue usada como polen padre, el porcentaje de amarre de frutos fue significativamente alto en 'Campas' $(85,83 \%)$ y 'White' $(87,40 \%)$. Además, tuvo un efecto significativo al incrementar el peso del fruto, número de semillas, peso de semillas, peso de pulpa y simetría en los 3 cultivares.

\begin{abstract}
Pollen source influence and effectiveness in the quality of Chirimoya fruits (Annona cherimola Mill.) The influence of the pollen source and the time of hand pollination on fruit set and fruit quality were evaluated in 3 cultivars of cherimoya: 'Campas', (Californ) 'White' and 'Bonita' (Spain). Artificial self-pollination and cross-pollination (pollen unmixed) were performed in each cultivar, at 3 different times of day (07:00-09:00, 10:00-12:00 and 18:0020:00 h), using the brush technique. In all cultivars, the percentage of fruit set with natural pollination was low (5 to 10\%). Self-pollination treatment was the less efficient, especially in 'Bonita' (47.90\%). Conversely, when 'Bonita' was used as pollen parent, fruit set percentage was significantly high in 'Campas' (85.83\%) and 'White' (87.40\%). Furthermore, the source of pollen had a significant effect, increasing fruit weight, number of seeds, seed weight, pulp weight and symmetry in the 3 cultivars.
\end{abstract}

Autor para correspondencia. Correo electrónico: acastanedav@uaemex.mx

Posgrado en Ciencias Agropecuarias y Recursos Naturales, Universidad Autónoma del Estado de México, Campus "El Cerrillo", El Cerrillo
Piedras Blancas, Toluca, Estado de México, C.P. 50200, México.

** Facultad de Ciencias Agrícolas, Universidad Autónoma del Estado de México, Campus "El Cerrillo", El Cerrillo Piedras Blancas, Toluca, Estado de México, C.P. 50200, México. 


\section{INTRODUCCIÓN}

La producción comercial de chirimoya (Annona cherimola Mill., Anonaceae) en México y otros países, está limitada por la ausencia de agentes polinizantes y una marcada dicogamia protogínica lo cual ha originado que el amarre o efectividad en la calidad de frutos producidos por polinización natural sea deficiente (Higuchi et ál. 1998, Duarte et ál. 2001, González y Cuevas 2008). Actualmente la técnica de polinización manual es practicada en países como Chile, España y Estados Unidos de Norteamérica, California donde ha mejorado el amarre de frutos y substancialmente ha incrementado el rendimiento y calidad (Duarte y Escobar 1997, Da Silva et ál. 2004, Lora et ál. 2006); sin embargo el amarre y desarrollo de los frutos es variable en flores polinizadas manualmente en un mismo árbol, que indican la existencia de otros factores que limitan la producción.

Entre estos factores esta la disponibilidad $\mathrm{y}$ fuente de polen, un factor potencial que determina la producción exitosa de frutos en plantas silvestres y cultivadas (Wallance y Lee 1999, Voyiatzis y Paraskevopoulou 2002). Como antecedente se sabe que la influencia de la fuente de polen sobre la semilla (xenia) y el fruto (metaxenia) es bien conocida en cultivos, además de las anonáceas (Denney 1992), para la cual (Kanh et ál. 1994) menciona que los efectos xenicos incluyen un amplio rango de diferencias en tamaño, forma, color, desarrollo y composición química de semillas y frutos.

Según lo anterior se plantea que la fuente de polen tiene influencia directa en la calidad de la masa y simetría del fruto. Por otra parte el número de pistilos polinizados exitosamente determinan la distribución y desarrollo de la semilla, la cual esta positivamente correlacionada con el crecimiento del fruto; además las semillas producen las fitohormonas auxinas y giberelinas, estimulantes de tejidos que incrementan la masa del fruto (Pritchard y Edwards 2006). Lo anterior, presume que la producción de frutos obtenidos a partir de polinización manual depende de la compatibilidad de la fuente de polen con la planta madre.

A partir del análisis anterior se consideró como objetivo de esta investigación la evaluación de la influencia de la fuente de polen sobre el amarre y calidad de frutos en 3 cultivares de chirimoya sometidos a diferentes tratamientos de polinización manual.

\section{MATERIALES Y MÉTODOS}

El estudio se llevó acabo en el huerto experimental de chirimoyos perteneciente al Centro Experimental "La Cruz" de la Fundación Salvador Sánchez Colín CICTAMEX, S.C., en Coatepec Harinas, Estado de México. El huerto se encuentra ubicado a $18^{\circ} 55^{\prime} 02.4^{\prime \prime} \mathrm{LN}, 99^{\circ} 45^{\prime} 31.8^{\prime \prime}$ LO y altitud de $2219 \mathrm{~m}$. Se seleccionaron 5 árboles de chirimoya de 15 años de cada uno de los siguientes cultivares, 'Campas', 'White' y 'Bonita' injertados sobre portainjertos criollos, plantados a una distancia de $5 \times 5 \mathrm{~m}$, podados a una altura de $2,0 \mathrm{~m}$ y con sistema de conducción de vaso abierto.

El cultivar 'White' se siembra comercialmente en California, mientras que 'Campas' y 'Bonita' son cultivados en menor escala en España.

Durante el periodo de floración (marzo a mayo) de 2011, los árboles seleccionados fueron polinizados manualmente bajo un diseño de bloques completos al azar con esquema factorial $3 \times 3 \times 3$. Se realizó autopolinización y polinización cruzada sin mezcla de polen en cada cultivar en 3 horarios diferentes: 07:00-09:00, 10:00-12:00 y 18:00-20:00 h.

La polinización manual se realizó con la técnica de pincel descrita por Schroeder (1946), donde flores en estado macho de los cultivares 'Campas' y 'Bonita' fueron recolectadas a las 16:00 h y las de 'White' a las 17:00 h, ya que durante esas horas las flores de cada variedad, se transforman de flor femenina a masculina y ocurre la liberación del polen. Para las pruebas, el polen fue extraído de flores en etapa masculina con ayuda de un pincel de pelo de camello y 
posteriormete se almacenó en recipientes de plástico de $5,0 \mathrm{~cm} \times 2,5 \mathrm{~cm}$ a temperatura ambiente. Se polinizaron 40 flores por tratamiento en el estado 61 (hembra) de acuerdo con la escala BBCH (Cautín y Agustí 2005); seleccionadas de forma aleatoria de la parte media y basal del árbol. Para esto, se sustrajo polen con un pincel fino de pelo de camello $(0,03 \mathrm{~g})$ y entre las aberturas de los petalos, se depositó sobre el cono estigmático por medio de 3 movimientos circulares en su superficie. Para evitar contaminación cruzada, se destinaron diferentes envases plásticos y pinceles para cada fuente de polen.

Las flores de cada tratamiento fueron etiquetadas con tiras plásticas de colores para su identificación y adicionalmente, se marcaron 50 flores sometidas a polinización natural en cada variedad. En el área experimental se colocó un higrotermómetro HOBO (Onset Computer Corporation, Bourne, MA, USA) que se utilizó para el registro de temperaturas y humedad relativa durante el desarrollo del experimento, con el propósito de determinar la influencia de estos factores en al amarre de frutos. El porcentaje de amarre de frutos fue determinado visualmente a los 15 días posteriores a la polinización manual, observándose pequeños agregados del fruto, a la vez, llevó a cabo un monitoreo con intervalos de 30 días con el fin de registrar el amarre final de frutos.

Entre noviembre de 2011 a enero 2012, se cosecharon y clasificaron los frutos fisiológicamente maduros de acuerdo con la escala sugerida por George et ál. (1992): 1 que corresponde a frutos deformes y $5=$ que corresponden a frutos muy simétricos. Se seleccionó una muestra al azar de 15 frutos y se llevaron al laboratorio donde se evaluaron variables biofísicas como: el peso del fruto $(\mathrm{g})$, peso de pulpa (g), peso de semillas por fruto $(\mathrm{g})$, número de semillas, peso medio de las semillas (g), índice de semillas (número de semillas en $100 \mathrm{~g}$ de pulpa). Dentro de las variables bioquímicas se estimó el contenido de solidos solubles totales (SST) expresados en ${ }^{\circ}$ Brix y el porcentaje de acidez titulable (AT).
Se realizó un análisis de varianza con el programa estadístico SAS ${ }^{\circledR}$ (SAS Institute 2002). Para la comparación de medias Tukey (alpha $=0,05)$ de las variables peso de fruto, número de semillas, peso semillas, índice de semillas, peso de pulpa. El porcentaje de amarre de frutos a la cosecha se comparó entre los diferentes tratamientos de autopolinización y polinización cruzada, con la prueba no paramétrica de Kruskal-Wallis, debido a que los datos transgreden supuestos básicos de normalidad.

Por último se correlacionó el número de semillas/peso del fruto y número de semillas/ simetría del fruto; la simetría de los frutos entre tratamientos se comparó por medio de la prueba de $X^{2}$.

\section{RESULTADOS Y DISCUSIÓN}

El porcentaje de amarre de frutos obtenidos a partir de la polinización natural fue considerado bajo pues mostró un rango entre 5 y $10 \%$ para los 3 cultivares. Estos resultados son similares a los obtenidos por (Kanh et ál. 1994, Richardson y Anderson 1996); y sugirieren que existe baja actividad de insectos polinizantes y naturaleza protogínica de las flores. Por su parte la fuente de polen empleada en polinización manual presentó diferencias significativas en el amarre de frutos en todo los cultivares (prueba Kruskal-Wallis $\mathrm{H}=73,23$; $\mathrm{p}=0,0001$ ). En los cultivares 'Campas' y 'White', el mejor porcentaje de amarre de frutos se obtuvo con la fuente de polen de 'Bonita' en un 85,83 y $87,40 \%$, respectivamente (Figura 1), por el contrario en 'Bonita' la fuente de polen más eficiente en el amarre de frutos fue la del cultivar 'Campas' con un 58,66\% (Figura 1) y además la interacción del cultivar con la fuente de polen fue significativa al $(\mathrm{p}<0,001)$.

Estos resultados son un indicativo de que el polen de 'Bonita' mejora significativamente el amarre e incrementa la producción de frutos, lo que sugiere que existe un mayor grado de compatibilidad cruzada entre los cultivares 'Campas' y 'White'. El incremento de amarre de frutos que se obtuvo con polinización cruzada coincide con 

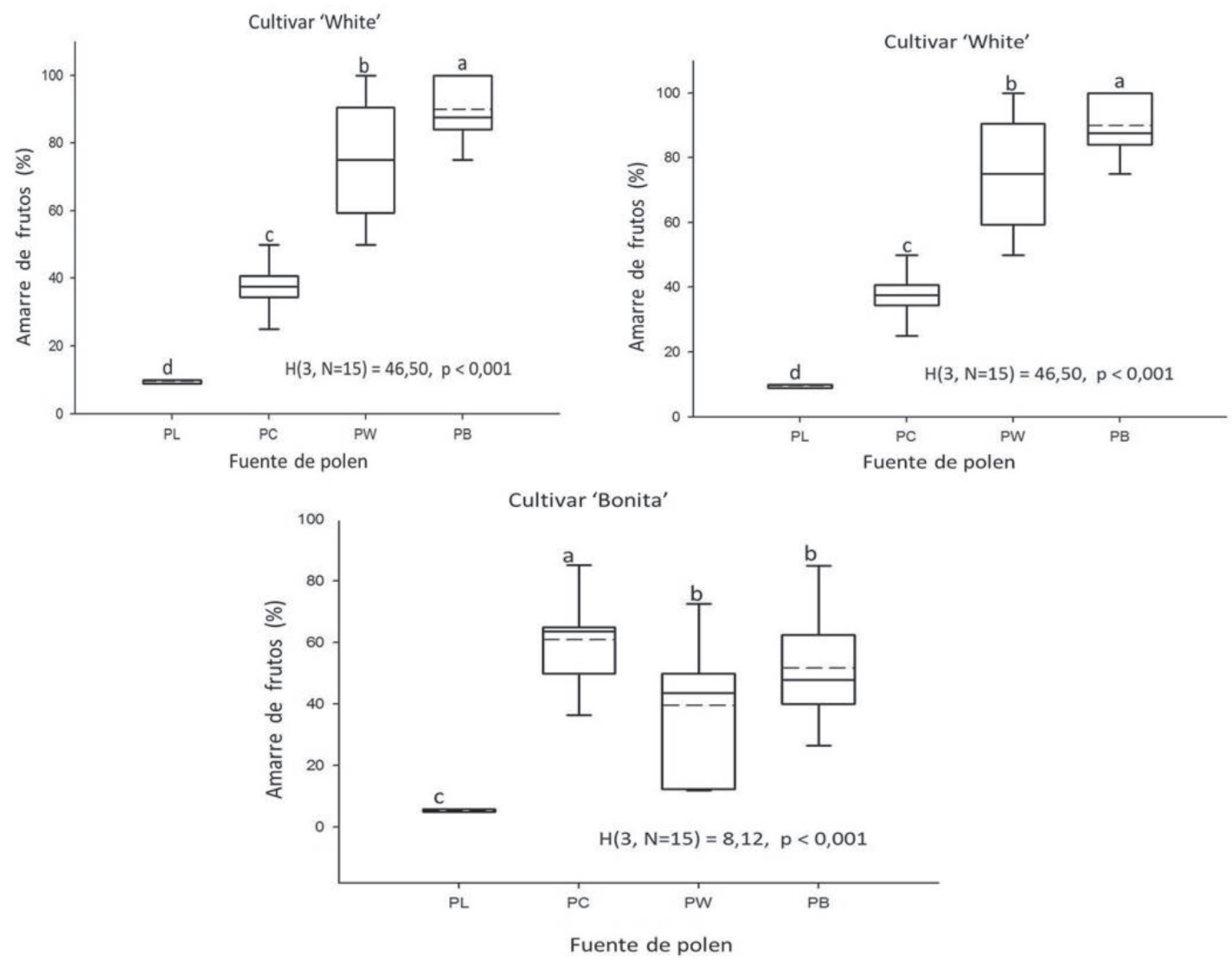

Fig. 1. Amarre de frutos en cv. 'Campas', 'White' y 'Bonita' obtenidos de la polinización manual con diversas fuentes de polen. [PL = polinización libre, $\mathrm{PC}=$ polen de 'Campas', $\mathrm{PW}=$ polen de 'White', $\mathrm{PB}=$ polen de 'Bonita' $]$. Cajas etiquetadas con la misma letra no son estadísticamente diferentes al $\mathrm{p}<0,05$; prueba de Kruskal-Wallis.

el obtenido por (Duarte y Escobar 1997), donde la polinización de chirimoya cultivar 'Cumbe' se incrementó con polen del cultivar 'Bronceada'. De igual forma (Rosa et ál. 2002), reportó un incremento en el amarre de frutos de atemoya cultivar 'Gefner' con polen de A. squamosa L. Estudios realizados por (Pritchard y Edwards 2006) sugieren que el número de óvulos fecundados de manera exitosa dependen de la fuente de polen, además indican que están relacionados con niveles hormonales. El horario de polinización también tuvo un efecto significativo $(\mathrm{p}<0,01)$ sobre el amarre de frutos.
En todos los cultivares, las flores polinizadas en la tarde (18:00-20:00 h) amarraron mayor número de frutos. Las temperaturas y humedad relativa registradas en este horario oscilaron entre $20,83^{\circ} \mathrm{C}$ a $21,16^{\circ} \mathrm{C}$ y $31 \%$ a $42 \%$, respectivamente (Figura 2). De igual forma, otras evidencias han mostrado que el amarre de frutos se incrementa cuando se poliniza con polen recién colectado (Richardson y Anderson 1996, Duarte et ál. 2001). Es posible suponer que las condiciones ambientales antes mencionadas permitieron una mejor adhesión de los granos de polen en los estigmas 


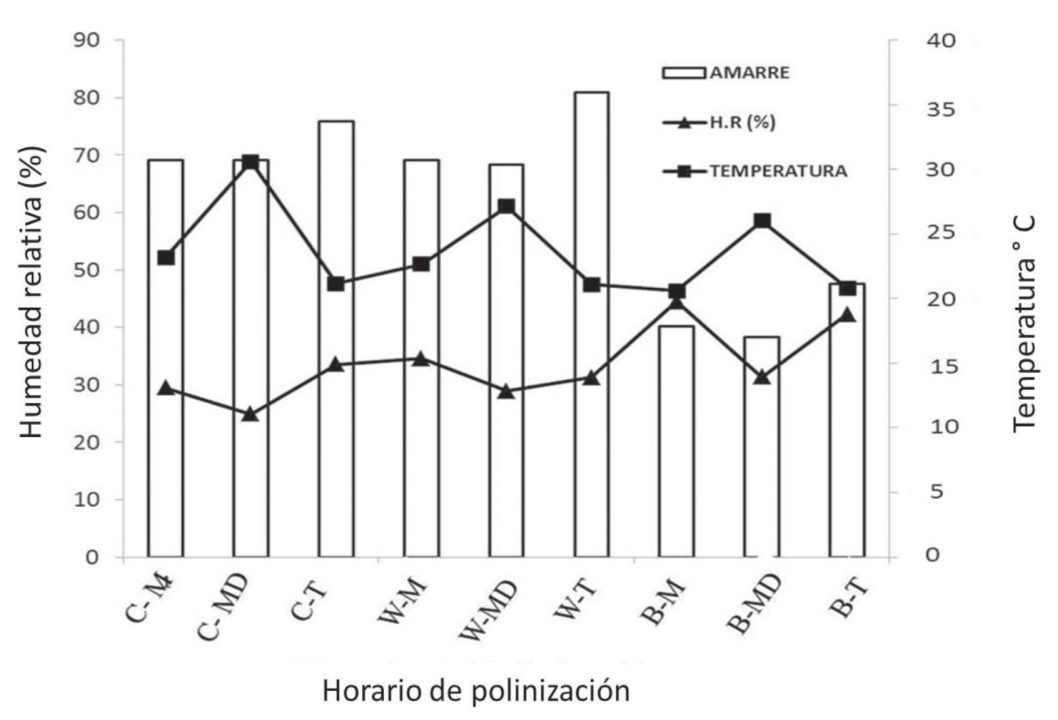

Fig. 2. Registro de temperatura y humedad relativa en diferentes horarios de polinización manual. $[\mathrm{C}=\mathrm{Campas}, \mathrm{W}=\mathrm{White}$, $\mathrm{B}=$ Bonita, $\mathrm{M}=$ mañana, $\mathrm{MD}=$ medio día, $\mathrm{T}=$ tarde $]$.

y evitaron su desecación. En este sentido, (Koura et ál. 2001, Lora et ál. 2006) reportaron que la chirimoya tiene un bajo amarre de frutos cuando las temperaturas oscilan entre $\operatorname{los} 30^{\circ} \mathrm{C} / 25^{\circ} \mathrm{C}$ debido a la baja viabilidad del polen y a la reducción del fluido viscoso en el cono estigmático. Por otro lado, Paull y Duarte (2011) mencionan que porcentajes de humedad relativa ambiental menores a $30 \%$ contribuyen a una pobre fecundación.

El efecto de la fuente de polen sobre el peso del fruto fue significativo al $(\mathrm{p}<0,01)$. En 'Campas', flores polinizadas con polen de 'Bonita' produjeron frutos de mayor peso; por otra parte, en 'White' los frutos más grandes se obtuvieron con polen de 'Bonita' (556,33 g) y 'Campas' (524,40 g). El menor peso de frutos se presentó en el cultivar 'Bonita' cuando se polinizo con el polen de 'White' (Cuadro 1). La diferencia en el peso medio de frutos entre los tratamientos refleja el número de semillas producidas de manera exitosa por la fuente de polen, ya que el número de semillas esta positivamente relacionado con el crecimiento del fruto debido a que las semillas producen fitohormonas (auxinas y giberelinas) que estimulan el desarrollo del fruto (Dag y Mizrahi 2005). Resultados similares fueron reportados por (Jalikop y Kumar 2007) quienes encontraron que el polen tiene un efecto significativo sobre el peso del fruto.

El número de semillas por fruto fue significativamente mayor en frutos obtenidos a partir de la polinización manual con el polen de 'Bonita' en los 3 cultivares (Cuadro 1), cuyos resultados muestran que el polen de esta variedad fecundó un mayor número de pistilos, lo que proveyó un mayor número de semillas. El número de semillas ha sido explicada por Lee 1988, quien señala que el número es bajo en frutos de plantas comerciales que han sido polinizadas con polen del mismo cultivar, aspecto que sugiere que existe una base genética que explica el aborto de las semillas. Además, se observó una correlación positiva y significativa $(\mathrm{p}<0,05)$ entre el número de semillas y peso del fruto en todos los cultivares (Cuadro 2). Resultados similares fueron encontrados en trabajos previos sobre chirimoya y el hÍbrido atemoya (Kanh et ál. 1994, Richardson y Anderson 1996, George et 
AGRONOMÍA COSTARRICENSE

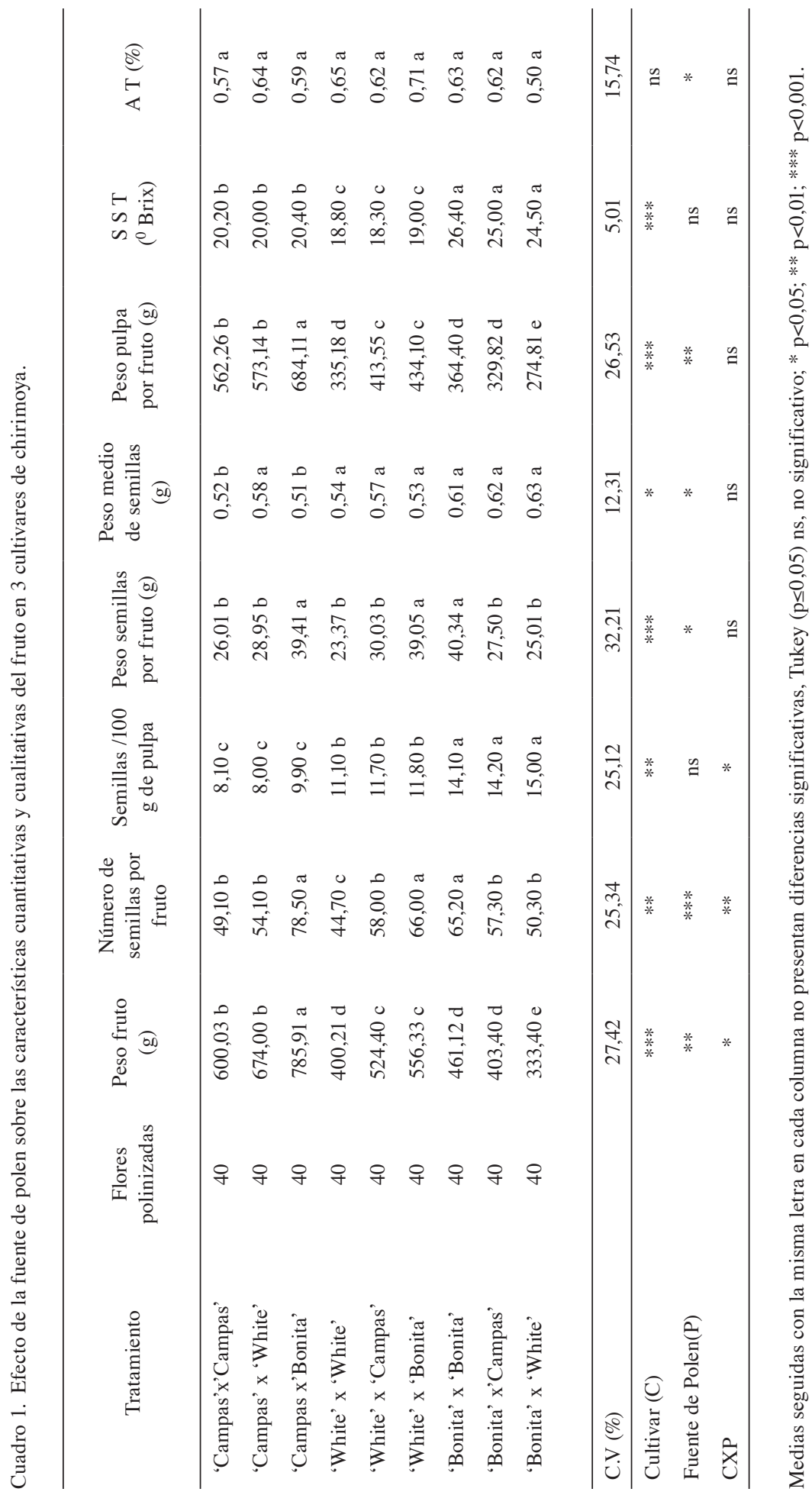

Agronomía Costarricense 39(1): 61-69. ISSN:0377-9424 / 2015 
ál. 2002, Pritchards y Edwards 2006, González y Cuevas 2008), indicando que a medida que aumenta el número de semillas el tamaño del fruto se incrementa. También se ha identificado que un número menor de semillas en la pulpa mejora la calidad consumible del fruto, pero para asegurar la simetría del fruto es necesario que un gran número de óvulos sean fecundados exitosamente y generen semillas que promuevan un buen desarrollo carpelar.

Cuadro 2. Coeficiente de correlación fenotípica entre las características de los frutos de los genotipos evaluados.

\begin{tabular}{llllllllc}
\hline Características & PF & NSF & IS & PS & PMS & PPF & SST & AT \\
\hline PF & 1,00 & $0,85^{*}$ & $0,83^{* *}$ & $-0,06 \mathrm{~ns}$ & $-0,67 \mathrm{~ns}$ & $0,98^{* * *}$ & $-0,27 \mathrm{~ns}$ & $-0,16 \mathrm{~ns}$ \\
NSF & & 1,00 & $0,13 \mathrm{~ns}$ & $0,72^{* *}$ & $-0,24 \mathrm{~ns}$ & $0,47 \mathrm{~ns}$ & $-0,21 \mathrm{~ns}$ & $0,23 \mathrm{~ns}$ \\
IS & & & 1,00 & $-0,85^{* *}$ & $0,63^{*}$ & $-0,90^{* * *}$ & $0,46 \mathrm{~ns}$ & $0,30 \mathrm{~ns}$ \\
PS & & & & 1,00 & $0,33 \mathrm{~ns}$ & $-0,03 \mathrm{~ns}$ & $0,52 \mathrm{~ns}$ & $0,54 \mathrm{~ns}$ \\
PMS & & & & & 1,00 & $0,69^{*}$ & $0,52 \mathrm{~ns}$ & $-0,34 \mathrm{~ns}$ \\
PPF & & & & & & 1,00 & $0,30 \mathrm{~ns}$ & $0,21 \mathrm{~ns}$ \\
SST & & & & & & 1,00 & $-0,96^{* *}$ \\
AT & & & & & & & & \\
\hline
\end{tabular}

${ }^{1}$ PF: peso del fruto; NSF: número de semillas por fruto; IS: índice de semillas; PS: peso de semillas; PMS: peso medio de semillas; PPF: Peso de pulpa por fruto.

El índice de semillas por fruto no se vio afectado por la fuente de polen dentro de un mismo cultivar. Sin embargo, el cultivar 'Campas' mostró un menor índice de semillas de 8,10 a 9,90 semillas/100g de pulpa. Por el contrario, a pesar de que el cultivar 'Bonita' tuvo un peso significativamente menor a los otros cultivares, presentó mayor índice de semillas de 14,10 a 15,0 semillas/100 g de pulpa (Cuadro 1), resultados similares fueron obtenidos por Richardson y Anderson 1996. El peso de semillas por fruto se vio afectado por la fuente de polen, en los 3 cultivares, aunque presentaron mayor peso cuando fueron polinizados con polen de 'Bonita'.

Una tendencia similar fue observada para el peso de pulpa, donde el polen de 'Bonita' en los 3 cultivares, 'Campas' (684,11 g), 'White' $(434,10 \mathrm{~g})$ y 'Bonita' (364,40 g), tuvo un efecto significativo (Cuadro 1). Además se observó una correlación negativa y significativa entre el peso de pulpa por fruto y el índice de semillas (Cuadro 2). Estos resultados son similares a los obtenidos en estudios previos sobre anonáceas (Jalikop y Kumar 2007) y en otras especies comerciales, como mandarina donde (Wallance y Lee 1999) mencionan que el peso de pulpa fue significativamente mayor cuando el cultivar 'Imperial' se polinizó con polen de 'Ellendal', 'Murcott' y 'Ellenor'.

El contenido de SST $\left({ }^{\circ}\right.$ Brix) y AT no se vio afectado por la fuente de polen. Sin embargo los valores más altos de SST se presentaron en el cultivar 'Bonita' (24,50 a 26,40 ${ }^{\circ} \mathrm{Brix}$ ) (Cuadro 1). Resultados similares fueron reportados por (Kanh et ál. 1994), el cual encontró que el polen usado como padre no afecto el nivel de SST y AT en frutos de chirimoya. Además se observó una correlación negativa y significativa $(\mathrm{p}<0,01)$ entre la AT y SST (Cuadro 2). La forma o simetría del fruto también se vio afectada por la fuente de polen. En los 3 cultivares el mayor número de frutos completamente simétricos con categoría 5 se obtuvieron al polinizarlos con polen de 'Bonita' $\left(X^{2}=27,55 \mathrm{gl}=8 ; \mathrm{p}=0,005\right.$; Figura 3). 


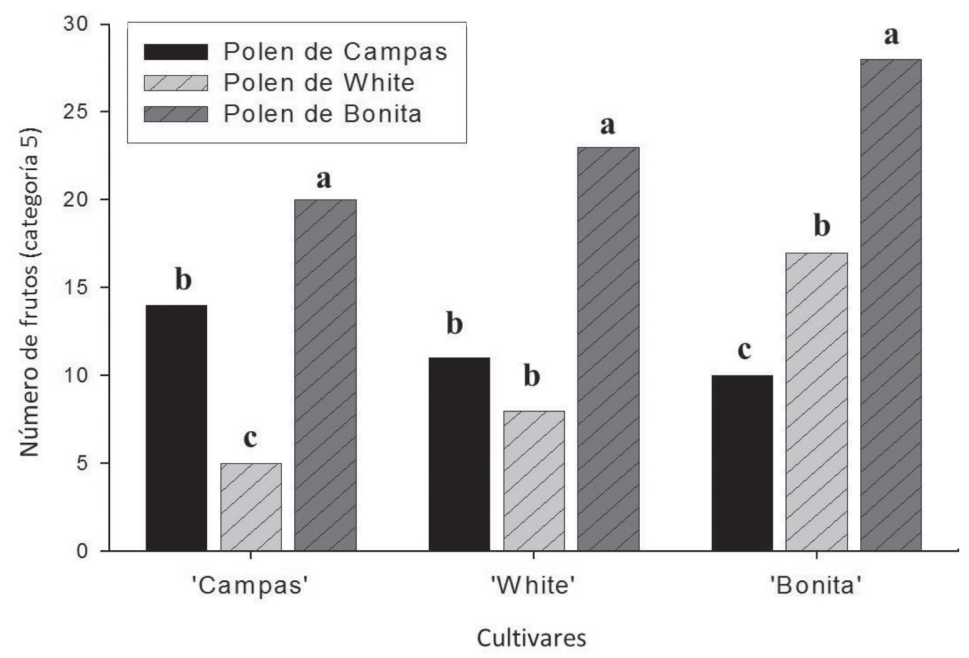

Fig. 3. Número de frutos dentro de la categoría 5 en 3 cultivares polinizados manualmente con 3 fuentes de polen.

La simetría es una característica que determina el valor comercial de frutos de chirimoya, debido a que frutos más grandes y simétricos tienen mayor aceptación en el mercado y además adquieren precio de mercado más altos. De acuerdo con los resultados en el presente estudio y otros estudios previos (Raven et ál. 1999, Pritchard y Edwards 2006), han demostrado una asociación positiva entre el número de semillas y simetría del fruto, lo que destaca que para lograr frutos con una simetría perfecta, el número y distribución de óvulos fecundados de manera exitosa juega un papel importante.

\section{CONCLUSIÓN}

La polinización de flores de chirimoya fue más efectiva inmediatamente después de la colecta de polen comparada con la polinización realizada en la mañana y medio día; dicho aspecto sugiriere que la viabilidad del polen es una gran limitante en la polinización. El amarre de frutos se incrementó con polinización cruzada más que con autopolinización, con la mejor fuente de polen a partir del cultivar 'Bonita'. De los 3 cultivares evaluados 'Campas' presentó mayor producción de frutos al ser polinizada con polen de 'Bonita'. Bajo las condiciones de estudio se puede concluir que la polinización manual es necesaria para incrementar la producción y calidad sensorial de los frutos y así como su simetría.

\section{AGRADECIMIENTOS}

El presente trabajo fue financiado en parte por la Secretaria de Educación Publica (SEP) a través del proyecto PROMEP /103.5/10/4368. Al consejo Nacional de Ciencia y Tecnología, por el financiamiento de los estudios de postgrado de Isela Apolonio Rodríguez y a la Fundación Salvador Sánchez Colín, CICTAMEX, S.C. por las facilidades otorgadas para la realización del trabajo experimental en el banco de Germoplasma de chirimoya. 


\section{LITERATURA CITADA}

CAUTÍN R., AGUSTÍ M. 2005. Phenological growth stages of the cherimoya tree (Annona cherimola Mill.). Scientia Horticulturae 105(4):491-497.

DAG A., MIZRAHI Y. 2005. Effect of pollination method on fruit set and fruit characteristics in vine cactus Selinecereus megalanthus ('yellow pitaya'). Journal of Horticultural Science \& Biotechnology 80(5):618622.

DA SILVA R., PINTO E.E., FIGUEREDO J., PEREIRA F.K., DIAS A., BARROS P.G. 2004. Polinização natural, manual e autopolinização no pegamento de frutos de pinheira (Annona squamosa L.) em Alagoas. Revista Brasileira de Fruticultura 26(2):261-263.

DENNEY J.O. 1992. Xenia includes metaxenia. HortScience 27(7):722-728.

DUARTE O., ESCOBAR O. 1997. Improving fruit set of cherimoya (Annona cherimola Mill.) cv. Cumbe, by autogamous and allogamous hand pollination. Proceedings of the Interamerican Society for Tropical Horticulture 41:162-165.

DUARTE O., PINEDA A., RODRÍGUEZ P.P. 2001. Mejora del cuajado en Atemoya 'Gefner' (Annona cherimola x Annona squamosa) con diversos tratamientos de polinización manual. Proceedings of the Interamerican Society for Tropical Horticulture 45:74-76.

GEORGE A.P., BROADLEY R.H., NISSEN R.J., HAMILL S.D., DREW R. 2002. Breeding new varieties of atemoya (Annona spp. Hybrids). Acta Horticulturae 575:323-328.

GEORGE A.P., NISSEN R.J., CAMPBELL J.A. 1992. Pollination and selection in Annona species (cherimoya, atemoya and sugar apple). Acta Horticulturae 321:178-185.

GONZÁlez M., BAeZA E., CUEVAS J. 2006. Pollen load affects fruit set, size, and shape in cherimoya (Annona cherimola Mill.). Scientia Horticulturae 110:51-56.

GONZÁLEZ M., CUEVAS J. 2008. Optimal crop load and positioning of fruit in cherimoya (Annona cherimola Mill.) trees. Scientia Horticulturae 115(2):129-134.

HIGUCHI H., UTSUNOMIYA N., SAKURATANI T. 1998. High temperature effects on cherimoya fruit set, growth and development under greenhouse conditions. Scientia Horticulturae 77(1-2):23-31.

JALIKOP S.H., KUMAR P.S. 2007. Pseudo-xenic effect of Allied Annona spp. Pollen in Hand Pollination of cv. 'Arka Sanh' [(A. cherimola x A. squamosa]. HortScience 42(7):1534-1538.

KANH T.L., ADAMS C.J., ARPAIA M.L. 1994. Paternal and maternal effects on fruit and seed characteristics in cherimoya (Annona cherimola Mill.). Scientia Horticulturae 59(1):11-25.

KOURA S., HASEGAWA K., SUZUKI H., YAMAMOTO Y., YONEMOTO. 2001. Influence of high temperature and amount of viscous fluid on surface of pistils on the fruit set of cherimoya (Annona cherimola Mill.). Japanese Journal of Tropical Agriculture 45(4):275-280.

LEE T.D. 1988. Patterns of fruit and seed production, pp. 179199. In: J.W.L. DOUST (Ed.). Plant Reproductive Ecology. Oxford University Press, Oxford, UK.

LORA J., PÉREZ O.M.A., FUNTEAJA P., HORMAZA J.I. 2006. Low temperature storage and in vitro germination of cherimoya (Annona cherimola Mill.) pollen. Scientia Horticulturae 108(1):91-94.

PAULL R.E., DUARTE O. 2011. Tropical Fruits. $2^{\text {nd }}$ edition, Vol. 1. CAB International, Reading, U.K. 400 p.

PRITCHARD K.D., EDWARDS W. 2006. Suplementary pollination in the production of custard Apple (Annona sp.) -The effect of pollen source. Journal of Horticultural Science \& Biotechnology 81(1):78-83.

RAVEN P.H., EVERT R.F., EICHHORN S.E. 1999. The Biology of Plants. W.H. Freeman and Company Publishers, New York, USA, 543 p.

RICHARDSON A.C., ANDERSON P.A. 1996. Hand pollination effects on the set and development of cherimoya (Annona cherimola Mill.) fruit in a humid climate. Scientia Horticulturae 65(4):273-281.

ROSA M., VALDEVINO C., KAVATI R. 2002. Polinização artificial da atemóia com diversas fontes de pólen comparada com a natural. Bragantia 61(3):231-236.

SAS Institute. 2002. JMP. Statistic made visual. SAS Inst. Inc. U.S.A.

SCHROEDER C.A. 1946. Hand pollination effects in the cherimoya (Annona cherimola Mill.). California Avocado Society Yearbook 26:94-98.

VOYIATZIS D.G., PARASKEVOPOULOU G. 2002. Factors affecting the quality and in-vitro germination capacity of strawberry pollen. Journal of Horticultural Science \& Biotechnology 77(2):200203.

WALLACE H.M., LEE. L.S. 1999. Pollen source, fruit set and xenia in mandarins. Journal of Horticultural Science \& Biotechnology 74(1):82-86.

Todos los derechos reservados. Universidad de Costa Rica. Este artículo se encuentra licenciado con Creative Common Reconocimiento-NoComercial-SinObraDerivada 3.0 Costa Rica. Para mayor información escribir a rac.cia@ucr.ac.cr 
